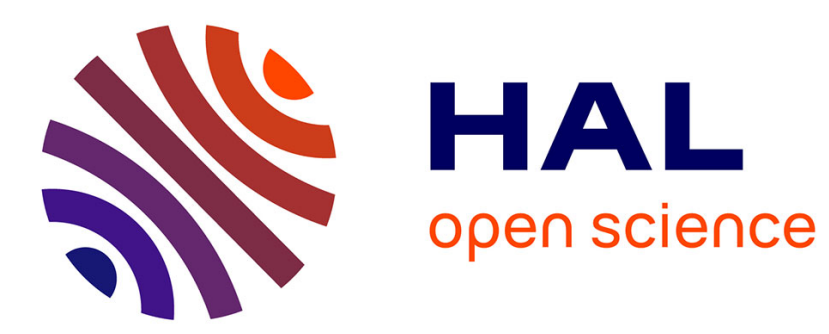

\title{
A la recherche d'une autre silviculture: l'individualisation précoce des arbres d'avenir
}

Marc de Saint-Vaulry

\section{To cite this version:}

Marc de Saint-Vaulry. A la recherche d'une autre silviculture: l'individualisation précoce des arbres d'avenir. Revue forestière française, 1969, 21 (2), pp.83-100. 10.4267/2042/20255 . hal-03385972

\section{HAL Id: hal-03385972 \\ https://hal.science/hal-03385972}

Submitted on 19 Oct 2021

HAL is a multi-disciplinary open access archive for the deposit and dissemination of scientific research documents, whether they are published or not. The documents may come from teaching and research institutions in France or abroad, or from public or private research centers.
L'archive ouverte pluridisciplinaire HAL, est destinée au dépôt et à la diffusion de documents scientifiques de niveau recherche, publiés ou non, émanant des établissements d'enseignement et de recherche français ou étrangers, des laboratoires publics ou privés. 


\title{
A LA RECHERCHE D'Une Autre Silviculture
}

\section{L'INDIVIDUALISATION PRÉCOCE DES ARBRES D'AVENIR}

\author{
M. de SAINT.VAULRY
}

Class. Oxford $181.6: 232.43$

Depuis plusieurs années, on constate une augmentation rapide du coût de la main-d'œuvre, il en résulte une diminution des prix des bois de faible diamètre. Les éclaircies deviennent de moins en moins rentables ef l'on se demande s'il ne faut pas augmenter l'intervalle entre deux éclaircies successives pour mieux les vendre. Ceci ralentirait la croissance en diamètre du peuplement. Or les diamètres importants sont justement recherchés spécialement dans les feuillus. On peut alors poser la question: la conduite de l'arbre en temps qu'individu dans un environnement de tiges support sans valeur, ne conduirait-elle pas à des résultats plus satisfaisants en meltant plus rapidement sur le marché des grumes de gros diamètres?

Ne doit-on pas concevoir la sylviculture en fonction du handicap de l'accrolssement raplde et constant du coût de la main-d'œuvre et de son remplacement, chaque fois que possible, par la mécanisation, afin de diminuer les prix de revient et d'augmenter le taux de placement du capital ?

\section{1 - PREA MBULE}

Dans un précédent article (1), nous avions évoqué l'avenir des bois de faible valeur destinés à la răperie. II semblait résider dans la mise en cuvre d'énormes machines réduisant au minimum le coût de la récolte; la concurrence des produits de remplacement étant telle qu'une augmentation du prix d'achat de la tonne de bois rendu usine reste aléatoire.

Le raisonnement s'étend logiquement de la monoculture de bois de râperie aux éclaircies des peuplements destinés à donner des grumes.

Le coût de ces produits de faible valeur reste limité par la concurrence des coûts de remplacement, la récolte pouvant difficilement être mécanisée comme dans le cas des monocultures, il y a impasse et on est logiquement conduit à penser que l'âge à partir duquel les éclaircies apporteront un bénéfice à leur propriétaire ne cessera d'augmenter. Parallèlement, à un âge donné, le bénéfice dû aux èclaircies ne cessera de diminuer. Par contre, les produits de gros diamètres semblent d'un avenir assurè.

Le revenu des éclaircies ne cessant de diminuer au profit de la récolte finale, nous pensons que les conditions économiques sont actuellement réunies ou en passe de l'être, à bréve échéance, pour qu'apparaisse une mutation fondamentale dans la sylviculture de fype traditıonnel.

(1) Revue Forestière Française, no 3, mars 1967. 
Si l'on considère les deux hypothèse suivantes, sur lesquelles tout le monde s'accorde:

- a longue échéance, le revenu des ventes des éclaircies tend à devenir nul,

$\rightarrow$ une grume de gros diamètre, bien élaguée, sans défaut, apte à donner du tranchage, du déroulage ou même du sciage de choix, a un débouché assuré dans l'avenir.

Ne faut-il pas reporter tout l'effort du sylviculteur sur l'éducation des arbres d'avenir (grosses grumes), au détriment des arbres à retirer en éclaircie (grumes de faible diamètre, peuplement de bourrage)?

La sylviculture du peuplement fait alors place a une éducation d'individus et on doit mettre au point une méthode d'individualisation précoce des arbres d'avenir.

Elle tend à individualiser les arbres le plus tôt possible et même dès la plantation.

Dans l'exposé qui suit, l'ensemble de la vie du peuplement de la plantation à la récolte, sera envisagé. Mais, dans une forêt, l'effort ne peut commencer à se concrétiser que sur des peuplements âgés de 0 à 70 ans. La récolte ne pouvant guère avoir lieu avant 100 ans et même 150 ans dans le cas du chêne, ceci implique pour les feuillus que les premiers arbres éduqués suivant cette méthode ne seront guère récoltés avant l'an 2000 , le coût de la main-d'œuvre aura alors trés fortement augmentè. La mécanisation se sera généralisée et le revenu des ventes des éclaircies de faible diamètre aura sans doute progressivement diminué, tandis que l'âge à partir duquel les éclaircies seront vendables aura augmenté.

Tout le raisonnement est donc basé - et il dolt l'être - sur la conjoncture économique de l'an 2000 , telle que nous essayons de la prévoir.

\section{2 - INDIVIDUALISATION PRÉCOCE DES ARBRES D'AVENIR}

\section{$2-1$ - DOMAINE D'EMPLOI}

2-1-1 - La méthode s'applique-t-elle à toutes les essences?

La plupart des essences forestières feuillues et résineuses sont concernées, tout en laissant à chacune ses caractéristiques propres.

La sylviculture, la variation de prix en fonction du diamètre et donc la hauteur de grume souhaitable, la rapidité de réponse à l'éclaircie, la précocité, la durée de vie, les utilisations, le diamètre envisageable de cime, la hauteur potentielle, tout varie d'une essence à l'autre.

La méthode doit donc s'adapter. Une éclaircie forte dans les pins, une fois la grume élaguée, n'ə que peu d'inconvénients. Par contre, une éclaircie trop forte d'un chêne maintenu serré serait catastrophique. La sylviculture du chêne pédonculè est différente de celle du rouvre (bien qu'ils soient souvent mélangés et traités de même). La différence de prix due au diamètre peut aller de 1 à 100 pour une grume de chêne, alors qu'elle ne dépasse guère 1 à 2 pour l'épicéa.

II est donc plus intéressant de faire des sacrifices pour l'obtention de gros diamètres chêne que pour de gros diamètres épicéas.

Si l'emploi de la méthode peut sembler moins utile pour certaines futaies telles que les saplnières, il pourrait être nécessaire pour la survie d'essences diverses actuellement menacées par la disparition du régime de taillis-sous-futaie (frêne, merisier, érable, noyer, etc.).

\section{2-1-2 - Ages d'application.}

Dans la conduite d'une futaie de type classique, il n'y a pratiquement jamais de choix d'arbres d'avenir. Tout arbre favorisé lors d'un martelage peut être défavorisé la fois suivante au profit d'un voisin mieux placé, mieux venant et moins taré. Ceci n'empêche pas bien entendu que certains beaux arbres soient " resélectionnés " à chaque martelage.

Dans la méthode employée à BERCE, les arbres sont individualisés entre 70 et 80 ans et leur choix n'est plus remis en cause.

La méthode d'individualisation précoce des arbres d'avenir s'appliquerait donc à la conduito des futaies d'âge inférieur à 70 ans.

- 40 à 60 ans : sélection définitive des arbres d'avenir feuillus.

- arbres de 25 à 30 ans: présélection des arbres d'avenir feuillus et sélection définitive de nombreux résineux.

- régénération acquise de 5 à 15 ans : ligne de sélection.

- régénération à faire : plantation d'arbres hautement sélectionnés dans un semis (ou une plantation) de peuplement de bourrage.

II pourra s'appliquer aussi à l'enrésinement des futaies feuillues et à la conversion des taillissous-futaies par sélection d'arbres d'avenir. 


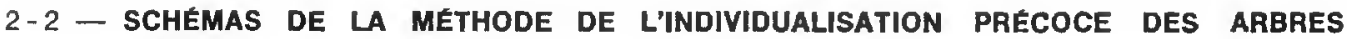 D'AVENIR}

En partant d'un peuplement dense, d'une régénération naturelle ou artificielle acquise, le but est d'obtenir une grume dont la hauteur sera prédéterminée par l'aménagiste en fonction de l'essence et de la richesse du sol, pour l'obtention rapide d'un gros diamètre.

Etudions donc une tige d'une essence quelconque prise dans un fourré d'essences identiques ou différentes.

Dans une première phase, cette tige doit s'allonger sans faire de branches de gros diamètre. Dès que les branches commencent à dépérir, à une hauteur qui correspond à celle de la grume désirée, le houppier est progressivement dégagé en tous sens fortement mais sans jamais être "isolé " jusqu'à ce qu'il atteigne la taille maximale permise par l'essence et le biotope (elle aussi prédéterminée par l'aménagiste).

Dans les premières années, le traitement est approximativement celui qui conduit à la futaie classique.

Les tiges sont serrées et le seul point où notre méthode diffère de la conduite classique d'un peuplement est la concentration du travail dans l'environnement immédiat de quelques tiges choisies, représentant une surface d'intervention restreinte par hectare et donc économique.

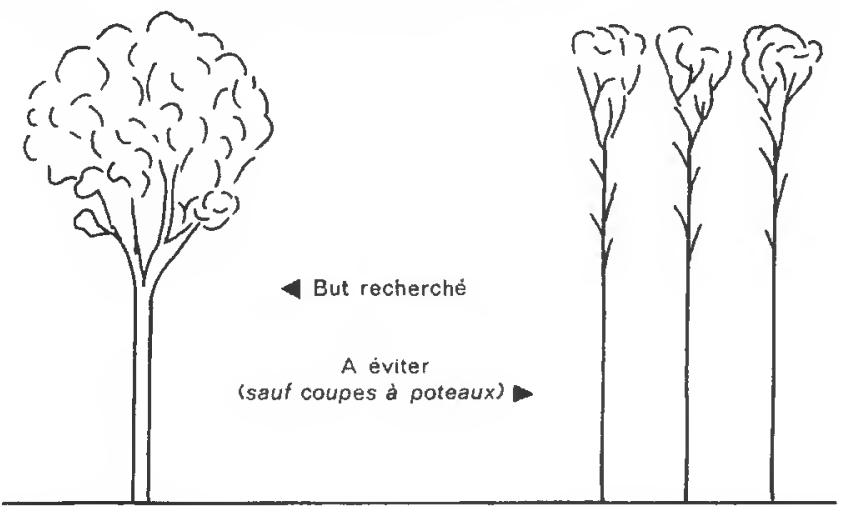

Dans la seconde phase, celle du développement du houppier, la méthode diffère de la conduite classique du peuplement en ceci qu'elle recherche une éclaircie également répartie tout autour de la cime de l'arbre choisi. Pour ce faire, le seul moyen est d'environner cette cime d'autres cimes nettement plus petites. Le reste du peuplement en dehors des arbres d'avenir, sera donc laissé à luí-même. A la fin du cycle, les cimes de tous les arbres d'avenir doivent être pratiquement en contact et le peuplement de bourrage doit être extrait en totalité (autant que faire se peut).

En aucun cas l'arbre d'avenir ne doit être "isolé ". L'intensité de l'éclaircie dépend toujours de la réaction possible de l'arbre (variable avec essence, richesse du sol, etc.).

Dans la méthode classique, les tiges d'avenir ne sont jamais définitivement choisles.

Dans la méthode proposée, le choix définitif se fait, suivant les terrains et les essences, de 35 à 60 ans pour les feuillus et de 20 à 35 ans pour les résineux, le choix provisoire pour les feuillus de 25 à 40 ans. Nous entendons par là, une présélection qui peut être remise en cause ultérieurement si nécessaire. En cas de changement, les arbres d'avenir sont pris dans le peuplement de bourrage, parmi les plus belles tiges de l'essence recherchée.

Enfin, dans les peuplements jeunes et en particulier dans les régénérations naturelles ou issues de semis, une présélection porte sur environ 1.000 arbres, repérés à l'aide de bandes tracées au gyrobroyeur (stade fourré) ou à la main (stade perchis). Seuls ces arbres font l'objet de dégagements de plantations et nettoiements. Lors de la sélection définitive des arbres d'avenir, ce choix pourra être remis en cause si certains sujets du peuplement de bourrage s'avéraient mieux venants.

Et enfin, dans les régénérations à faire, nous tendons vers une sélection génotypique avec plantation de 1.000 plants environ, hautement sélectionnés, dans une régénération " support * couvrant le sol de tiges non obligatoirement sélectionnées génétiquement.

Les arbres d'avenir seront alors obligatoirement pris dans ces 1.000 tiges. II peut en effet se faire qu'un arbre de remplissage semble supérieur aux arbres plantés. Rien ne prouve qu'il ne s'agisse pas là d'un "feu de faille "et que la production de ce dernier sera supérieure en fin de vie. 


\section{3 - DEVELOPPEMENT ET JUSTIFICATION}

\section{3-1 - LA SELECTION PRECOCE des tIges N'est-elle pAS aleAtoire?}

II n'est bien entendu pas possible de répondre que la sélection jeune est meilleure que la sélection d'arbres plus âgés. Néanmoins, et ceci nous semble fondamental, la sélection telle qu'elle est pratiquée actuellement d'arbres de 60 à 80 ans peut faire l'objet d'une erreur. En effet, outre le fait que cette sẻlection est phénotypique, elle conduit à sélectionner des arbres rustiques et résistants à la concurrence.

Prenons un exemple en zootechnie : mettons dans un enclos très étroit 10 vaches Salers rustlques et 10 vaches normandes, à haute capacité de production viande et lait, la quantité de nourriture ne permettant guère qu'à 3 ou 4 d'entre elles de se nourrir à peu près convenablement. Laissons-les pendant deux ans et venons voir les quelques survivantes. II est fort probable que les Salers résisteront mieux que les normandes moins rustiques. Le raisonnement de l'éleveur serait alors de dire en voyant 6 vaches Salers en relativement bon état et une vache normande en piteux état et sans doute atteinte de maladie: la race Salers est la meilleure, elle a fait ses preuves, dorénavant je n'élèverai plus que des vaches de race Salers. N'est-ce pas le raisonnement du forestier qui laisse s'exercer une concurrence sans mercl entre les tiges d'un peuplement d'une régénération où parfois, au départ, il y avait plus de 100 semis au $\mathrm{m}^{2}$ ?

Les tiges ayant le plus bel aspect sont, à notre avis, des tiges rustiques et résistant mieux à la concurrence. Ceci n'implique pas bien entendu qu'elles ne soient pas productives, mais force est de constater dans le monde vivant que souvent les rustiques sont moins productifs que les sélectionnés et qu'entre deux herbes la mauvaise, qui l'emporte souvent si on les laisse en concurrence, aurait été surpassée par une autre meilleure, si l'on avait donné à chacune sa chance (eau, matières minèrales, etc.).

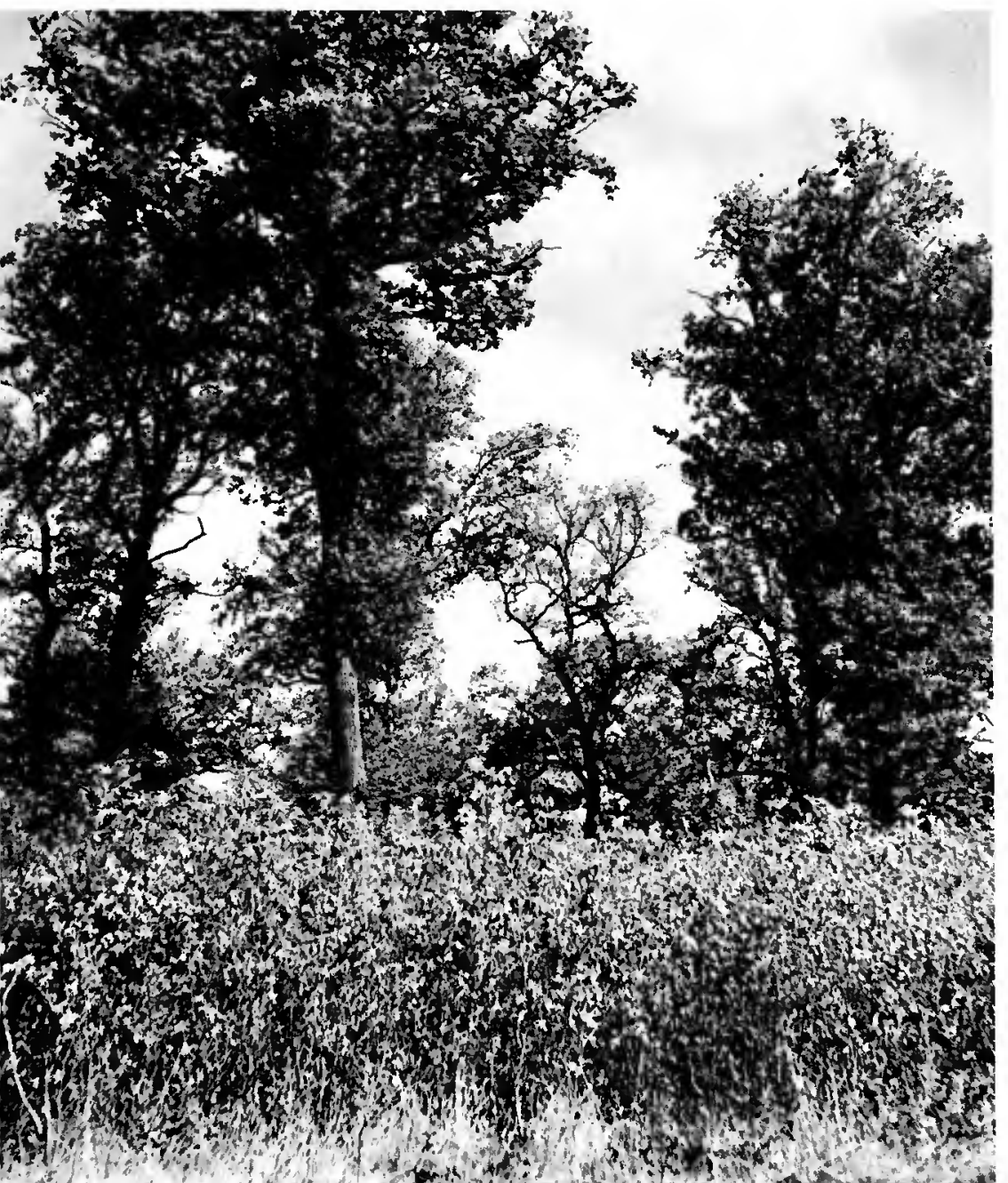

100 plants par $m 2$. La Jutte pour la survie, al elle conduit à un bon élagage naturel, n'est sans doute pas bonne pour la production quantitative. II n'est pas sür que les arbres ne "se souvlennent pas" de leur jeunesse difficile.

Ne conduit-elle pas à la sélection d'arbrea rustiques et resistant à la concurrence avec élimlnation posalble d'arbrea gros producteurs mais Inaptes à la concurrence? 
Sans bien entendu mettre en doute la valeur de la sélection traditionnelle, nous en concluons qu'une sélection précoce d'un certain nombre de tiges parmi les mieux venantes, sl elle permet aux dites tiges d'être mises plus tôt dans de meilleures conditions, a toute chance d'étre bénéfique pour la récolte, sans pour autant être systématiquement condamnée pour des considérations génétiques.

\section{3-2 - PEUT-ON DÉterminer d'APRÉs Le biotope, la hauteur souhaitable de GRUMES ?}

Actuellement les belles billes des foréts du Centre ne comportent guère plus de 3 à $5 \mathrm{~m}$ de tranche, rares sont celles qui peuvent en donner 10 à $12 \mathrm{~m}$. Le prix de la bille comportant quelques défauts chute rapidement.

La partie haute des grumes de relativement faible diamètre ne se vend pas cher. Pourquoi alors ne pas chercher à obtenir des grumes de gros diamètre et supprimer les surbilles ? Les deux sont étroitement liées; en effet, si l'on désire obtenir une grosse grume, Il faut un houppier important et dense; si la grume est trop longue, il ne peut être question d'avoir un houppier important.

L'aménagiste doit donc déterminer, en fonction de la conjoncture et de l'essence, la hauteur de grumes qui, tout en restant suffisante, permettra au houppier de se développer suffisamment pour la nourrir. Cette hauteur sl elle est caractérlstlque d'une essence doit aussl être adaptée à la richesse potentielie du sol (par richesse potentielle du sol nous entendons la richesse du sol au sens large et en particulier ce qu'elle deviendra après assainissement du terrain, travaux divers et apports d'engrais éventuels, compte tenu bien entendu des impératifs géologiques et climatiques). La hauteur de grume sur sol pauvre doit être plus faible que sur sol riche.

Nous préférons ne faire qu'une grume de 10 mètres et de $80 \mathrm{~cm}$ de diamétre plutôt que de faire dans le même temps 7 grumes de 15 métres et de $30 \mathrm{~cm}$ de diamètre, l'écart des prix au $\mathrm{m}^{3}$ pouvant étre de 1 à 10 , voire plus.

Le tableau page 9 donne une indication sur les hauteurs de grumes en fonction de la hauteur potentielle que doit atteindre l'arbre. Cette hauteur potentielle est à prendre au sens large car elle est appelée à varier pour un même so! suivant le traitement (taillis-sous-futaie, futaie, etc.), et les améliorations apportées artificiellement au sol.

Néanmoins, tout sylviculteur peut dire, en passant dans une friche calcaire sèche d'un plateau à végétation rabougrie, que la hauteur potentielle y a peu de chance d'atteindre 30 mètres alors que, dans une forêt bien drainée sur limon, elle a toute chance de les atteindre. Entre les deux, la hauteur potentielle est une notion qui nécessite une certaine culture forestière pour conduire à des chiffres...

\section{3-3 COMMENT DÉTERMINER LE DIAMEtTRE FINAL DE LA CIME?}

Ce diametre varie en fonction de l'essence, du mode de traitement et très probablement aussi de la richesse du sol.

\section{3-3-1 - Variation en fonction de l'essence}

Le sylviculteur reconnaitra souvent d'un simpie coup d'œil l'essence à la silhouette de l'arbre. Le port d'un laricio n'est pas le même que celul d'un pin noir et le port d'un hêtre diffère de celui du chêne. Pour chaque essence, il est possible de se fixer une silhouette d'arbre aptimale aprés de nombreuses observations sur le terrain dans ce but.

Une fois cette silhouette acquise, nous proposons pour obtenir des chiftres, de mettre la silhouette à l'échelle voulue à de légères modifications près. Dans les sols très pauvres, survant les essences, elle ne dépassera pas 7 a 15 mètres; dans les sols très riches, elle atteindra et même dépassera 25 mètres (chêne pédonculé). La hauteur de grumes et le diamètre du houppier dépendront donc de la hauteur de l'arbre (cette méthode permet de cerner le problème d'assez près, mais nous avons déjà vu des arbres de 10 mètres de haut et de plus de 20 mètres de diamètre de cime avec une grume de 3 mètres qui ne correspondaient bien entendu pas à la silhouette retenue). 


\section{3-3-2 - Variation en fonction du mode de traltement}

Le schéma ci-dessous montre schématiquement l'aspect de la cime d'un feuillu en fonction du mode de traitement.

1

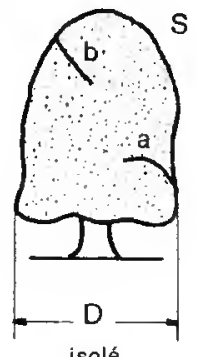

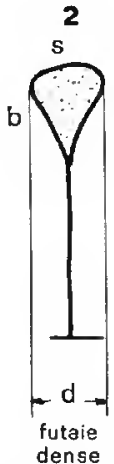

3

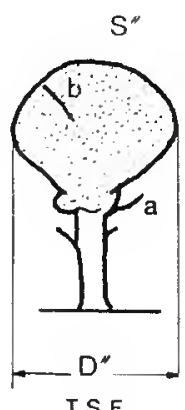

T.S.F.

D : Projection au sol du houppier (diamètre).

S: Surface foliaire extérieure exposée à la lumière.

a : Branche ayant commencé à pousser environ à 25 ans.

b : Branche ayant commencé à pousser environ à 70 ans.

Essayons de rechercher une explication du modelage de la cime dans chaque cas.

Figure 1: arbre de parc lsolé. La surface $S$ est très grande; par contre, la branche "a " ayant été dès sa naissance à l'action du soleil n'a pas cherché à s'allonger (1) et demeure relativement courte bien que développée. Le diamètre " $D$ " bien qu'important n'est pas forcément très grand puisqu'approximativement le double de la longueur de la branche * a w.

Flgure 2: arbre de futaie dense. L'arbre, maintenu serré, a vu sa branche " a " disparaître, sa premiére branche vive est la branche " $b$ m née tardivement. Elle n'a donc pas eu le temps de s'étendre.

Figure 3 : arbre de talliis-sous-futaie. L'arbre est soumis à des variations d'éclairement dans sa partie basse alors que sa cime est constamment maintenue au soleil.

Chaque coupe de taillis fait démarrer autant les branches basses que la cime. Son diamétre $D^{\prime \prime}$ sera assez important mais sa cime $S^{\prime \prime}$ sera moins importante que celle d'arbres de parc puisqu'elle descend moins bas.

Figure 4: arbre Individualisé jeune. La branche "a "a commencé a croitre lorsque l'arbre était jeune. Elle a toujours été à la limite de l'éclairement et donc a cherché à s'étendre d'abord en longueur, puis ensuite le houppier a cessé de croître en hauteur et sous l'effet des dégagements et du poids des semences s'est épanoul (particulièrement net chez le hêtre). Cette branche longue qui s'épanouit conduit à un diamètre $D^{\prime}$ qui doit très probablement être le plus grand des quatre tigures. Par contre, la surface $S^{\prime}$ n'est pas forcément aussi élevée que $S$. Toutes ces aftirmations constituent un schèma car s'il est possible de dire que la surface de l'arbre de futaie dense est faible, il est assez difficile d'être affirmatif en ce qui concerne le parallèle entre les trois autres. Mals il semble que le diamètre que l'on est en droit d'espérer sera très grand pour le cas 4.

\section{SCHÉMA DE FAÇONNAGE DU HOUPPIER}

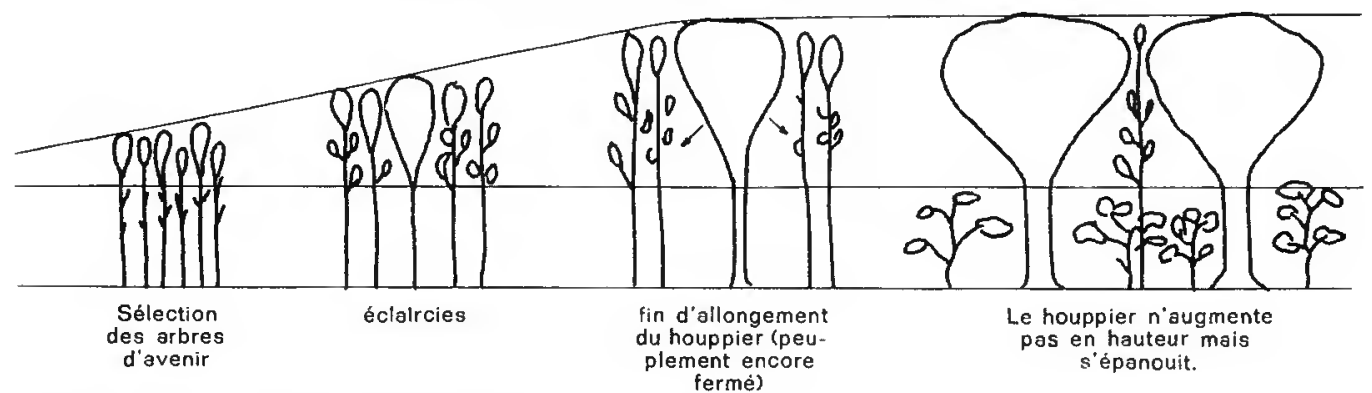

(1) Cette interpretation reste très discutable. 
Le tableau ci-dessous indique quels pourraient être à notre avis, les chiffres les plus probables pour chaque essence en fonction de la hauteur potentielle. Celle-ci est couramment de $25 \mathrm{~m}$. Pour les forêts les plus riches sur limon bien drainé, elle peut dépasser $30 \mathrm{~m}$.

NORMES APPROXIMATIVES

hauteur - clme - grume - densité/ha

\begin{tabular}{|c|c|c|c|c|}
\hline & $\begin{array}{l}\text { Hauteur } \\
\text { totale }\end{array}$ & $\begin{array}{c}\text { Largeur cime, } \\
\text { distance entre } \\
\text { pieds " }\end{array}$ & $\begin{array}{l}\text { Nombre de } \\
\text { pieds par ha } \\
\text { (au triangle) }\end{array}$ & $\begin{array}{l}\text { Hauteur } \\
\text { bille } \\
\text { de pied }\end{array}$ \\
\hline Chêne pédonculé ....... & $\begin{array}{l}30 \mathrm{~m} \\
25 \mathrm{~m} \\
20 \mathrm{~m}\end{array}$ & $\begin{array}{l}18 \mathrm{~m} \\
16 \mathrm{~m} \\
14 \mathrm{~m}\end{array}$ & $\begin{array}{l}35 \\
45 \\
60\end{array}$ & $\begin{array}{rl}11 & \mathrm{~m} \\
9 \mathrm{~m} \\
7 \mathrm{~m}\end{array}$ \\
\hline Chêne Rouvre $\ldots . . . \ldots$ & $\begin{array}{l}30 \mathrm{~m} \\
25 \mathrm{~m} \\
20 \mathrm{~m}\end{array}$ & $\begin{array}{l}15,5 \mathrm{~m} \\
14 \mathrm{~m} \\
12 \mathrm{~m}\end{array}$ & $\begin{array}{l}50 \\
60 \\
80\end{array}$ & $\begin{array}{r}12 \mathrm{~m} \\
10 \mathrm{~m} \\
7 \mathrm{~m}\end{array}$ \\
\hline Hêtre . .............. & $\begin{array}{l}30 \mathrm{~m} \\
25 \mathrm{~m} \\
20 \mathrm{~m}\end{array}$ & $\begin{array}{l}17 \mathrm{~m} \\
14 \mathrm{~m} \\
11 \mathrm{~m}\end{array}$ & $\begin{array}{r}40 \\
60 \\
100\end{array}$ & $\begin{aligned} 11 \mathrm{~m} \\
9 \mathrm{~m} \\
7 \mathrm{~m}\end{aligned}$ \\
\hline Charme . ............ & $\begin{array}{l}25 \mathrm{~m} \\
20 \mathrm{~m}\end{array}$ & $\begin{array}{l}8 \mathrm{~m} \\
6 \mathrm{~m}\end{array}$ & $\begin{array}{l}180 \\
320\end{array}$ & $\begin{array}{l}7 \mathrm{~m} \\
6 \mathrm{~m}\end{array}$ \\
\hline Frêne...$\ldots \ldots \ldots \ldots$ & $\begin{array}{l}30 \mathrm{~m} \\
25 \mathrm{~m} \\
(20 \mathrm{~m})\end{array}$ & $\begin{array}{l}18 \mathrm{~m} \\
15 \mathrm{~m} \\
(11 \mathrm{~m})\end{array}$ & $\begin{array}{c}35 \\
50 \\
(100)\end{array}$ & $\begin{array}{rl}10 & \mathrm{~m} \\
8 \mathrm{~m} \\
(6 \mathrm{~m})\end{array}$ \\
\hline Epicéa .............. & $\begin{array}{l}30 \mathrm{~m} \\
25 \mathrm{~m} \\
20 \mathrm{~m}\end{array}$ & $\begin{array}{l}8 \mathrm{~m} \\
7 \mathrm{~m} \\
6 \mathrm{~m}\end{array}$ & $\begin{array}{l}180 \\
235 \\
320\end{array}$ & $\begin{aligned} 14 \mathrm{~m} \\
12 \mathrm{~m} \\
9 \mathrm{~m}\end{aligned}$ \\
\hline Pin sylvestre Douglas ... & $\begin{array}{ll}30 & \mathrm{~m} \\
25 \mathrm{~m} \\
20 \mathrm{~m}\end{array}$ & $\begin{array}{rl}11 & \mathrm{~m} \\
8 \mathrm{~m} \\
7 \mathrm{~m}\end{array}$ & $\begin{array}{l}100 \\
182 \\
240\end{array}$ & $\begin{array}{rl}14 & \mathrm{~m} \\
12 \mathrm{~m} \\
9 \mathrm{~m}\end{array}$ \\
\hline
\end{tabular}

- Le pourcentage de couvert étant supposé de $100 \%$.

\section{3-3-3 - Incidence de la richesse du sol}

Actuellement, tout le monde s'accorde à dire que la hauteur des arbres traités avec une même méthode est un indice direct de la fertilité des sols.

Son incidence sur le diamétre du houppier est moins certaine. Mais pour s'en rendre compte, il suffit néanmoins de passer d'une futaie vigoureuse à une futaie " maigre "pour sentir que la richesse du sol doit influer sur le diamétre du houppier.

\section{3-3-4 - Diamètre tinal de la clme (densité par hectare)}

Après avoir analysé les différents facteurs, la hauteur potentielle de l'arbre étant déterminée, on obtient le diamétre de houppier et la hauteur correspondante de grumes, d'après les silhouettes. Il en découle le nombre d'arbres par hectare sachant que tout sera mis en œuvre pour obtenir une cime large. Le nombre d'arbres d'avenir à l'hectare est très bas. II Importe, une fols le nombre de pieds par hectares déterminé, de s'y fixer et de ne prendre en aucun cas des tiges de remplacement. 
II n'est pas question en effet, si on désire choisir 70 arbres d'avenir par hectare, d'en sélectionner 115, se disant qu'en cas de chablis il y aura des remplaçants. En effet, le chiffre de 70 correspond à un diamètre d'environ $13 \mathrm{~m}$ de cime (au triangle) et celui de 115 à $10 \mathrm{~m}$. Si 4 arbres étaient à extraire du fait de maladies avec chablis, seuls ses voisins atteindraient une taille correcte et tout le reste de la parcelle ne pourrait dépasser les $10 \mathrm{~m}$ de diamètre de cime qui ne correspondent pas au diamètre de grumes recherché.

Le schéma ci-dessous montre ce qu'il advient lorsque l'arbre d'avenir est à extraire. Dans un premier cas il est à extraire peu de temps après le début de la sélection. II n'a donc pas encore pris beaucoup d'avance sur les arbres voisins et l'un de ceux-ci parmi les plus vigoureux peut le remplacer sans difficulté.

Il donnera un tronc excentré correspondant à une cime dissymétrique (cas A).

Si l'arbre d'avenir est à extraire aux deux tiers de sa vie, il pourra dans certains cas donner une grume déjà appréciable et il reste encore des arbres de bourrage parmi lesquels il serait possible de choisir 1,2 ou 3 sujets de remplacement qui ne donneront alors que des grumes de petits diamétres (cas B).

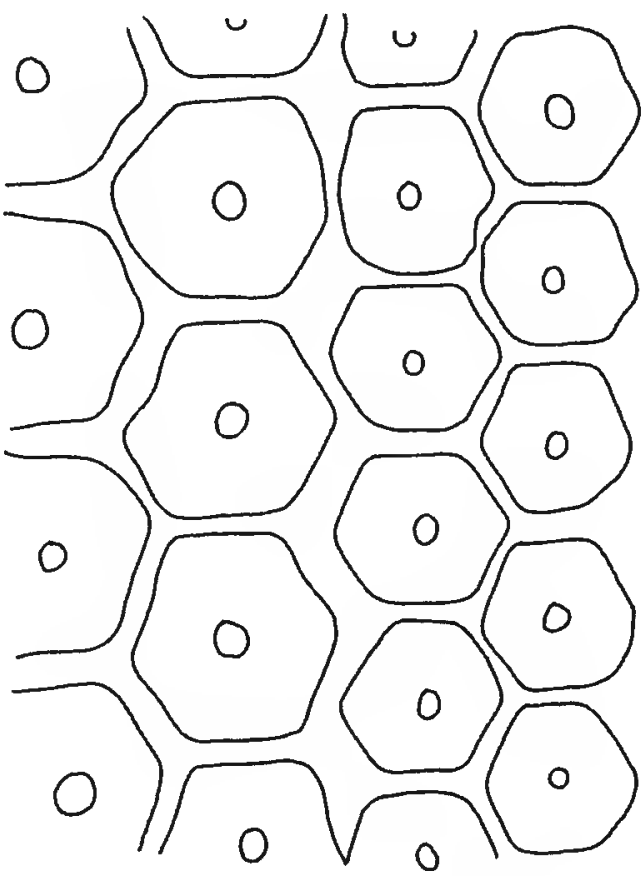

Arbres à bonne distance

Arbres trop serrés

\section{REMPLACEMENT DES ARBRES D'AVENIR PAR SUITE D'ACCIDENTS}

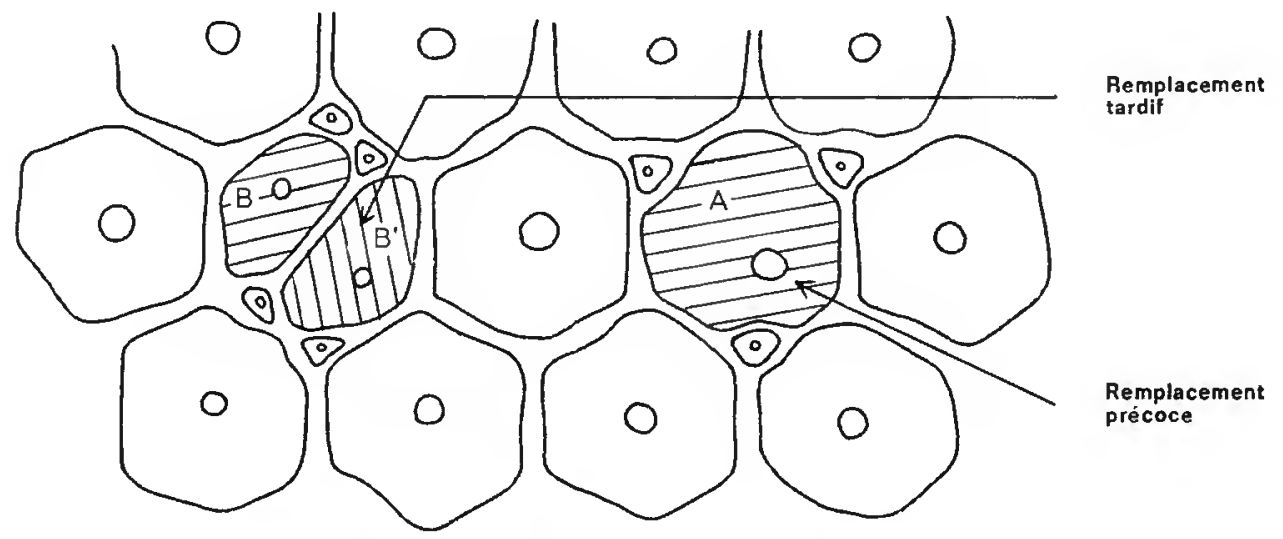

\section{3 - 4 - QUEL DIAMETRE DE GRUME EST-ON EN DROIT D'ATTENDRE ?}

Le calcul schématique est le suivant : si un hectare produit $4 \mathrm{~m}^{3}$ par an, dans des conditions d'éclaircie normale, le demi-hectare produira moitié moins, le dixième d'hectare dix fois moins et le mètre carré 10.000 fois moins.

En première approximation, il est donc possible de calculer le cube de bois produit par l'arbre d'avenir en intégrant la somme des surfaces couvertes par sa cime durant toute sa vie. 
Supposons par exemple qu'à 20 ans le diamètre de sa cime soít de $1 \mathrm{~m}$, sa production annuelle sera alors de $4 / 10.000 \mathrm{de}^{3}$. Quand sa cime atteindra un are, sa production sera de:

$$
\frac{4 \times 100}{10.000}=0,04 \mathrm{~m}^{3} / \mathrm{an} \text {. }
$$

La somme de ces différents chiffres donne le volume de l'arbre lors de la coupe.

Sachant que 45 à $50 \%$ de la production donnera du bois de chauffage, le reste sera donc une grume. $\mathrm{Si}$ la hauteur de grume est de 10 métres, on peut donc en déduire approximati vement son diamètre.

\section{COURBES DE CROISSANCE}
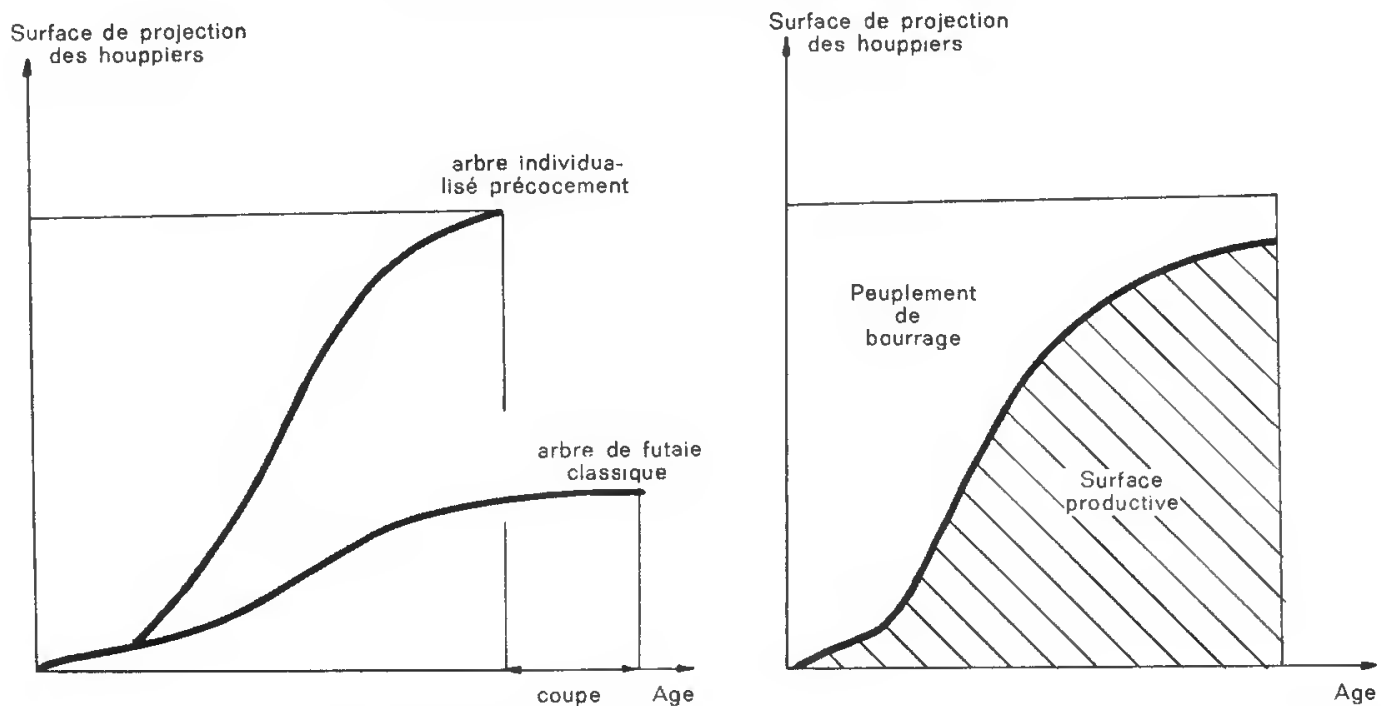

Ceci n'est juste qu'en premiére approximation car deux facteurs interviennent.

10 La production à l'hectare varie suivant l'âge du peuplement, la production annuelle étant maximale quand le peuplement est encore assez jeune. Ceci est encore en faveur de la méthode car dès ce moment, nous permettons à l'arbre de produire le maximum de bois grume.

$2^{0}$ Le fait d'avoir une cime ample conduit à une production de semences importante. Cela doit être au détriment de la production bois, et serait en défaveur de la méthode, mais pour un certain nombre d'essences les gelées et autres éléments interviennent pour limiter les floraisons. D'autre part, la production importante de semences devient intéressante au moment de la régénération.

\section{3-5 - ECLAIRCIES}

Les éclaircies dolvent être mesurées et non massives. Elles ne doivent pas conduire à des descentes de cimes, chez le chêne en particulier. Pour cela les premières seront rapprochées dans le temps. Le peuplement de bourrage restant serré, l'éclaircie pourra être plus forte autour de l'arbre d'avenir que dans l'éclaircie classique tout en étant moins forte sur l'ensemble du peuplement. Elle n'est plus uniforme.

Elles seront faites au minimum de prix pendant toute la durée du peuplement. Elles seront adaptées au pouvoir de réponse de chaque essence et de chaque âge. Nous croyons qu'aucune ne sera vendable. Par contre, et nous tenons à le préciser, l'accroissement rapide du diamètre de la grume permettra d'obtenir des produits finals, alors que, dans les méthodes traditionnelles de conduite de peuplement, on n'obtient encore que des produits d'éclaircie. Supposons par exemple pour le chêne que les cernes soient de $4 \mathrm{~mm}$ au lieu de 2, l'arbre pourrait être obtenu en moitié moins de temps.

Compte tenu des 30 à 50 années oủ le peuplement est maintenu serré on pourrait donc dire que, pour atteindre par exemple $60 \mathrm{~cm}$ de diamètre, s'il faut 150 ans avec des méthodes traditionnelles, notre méthode permettrait de l'obtenir environ à 100 ans, date à laquelle il y a encore peu d'éclaircies rentables de fait dans les peuplements de chêne. 
Par contre, si l'arbre est maintenu jusqu'à 150 ans, son diamètre pourrait théoriquement avoisiner $100 \mathrm{~cm}$ de diamètre au lieu de $60 \mathrm{~cm}$.

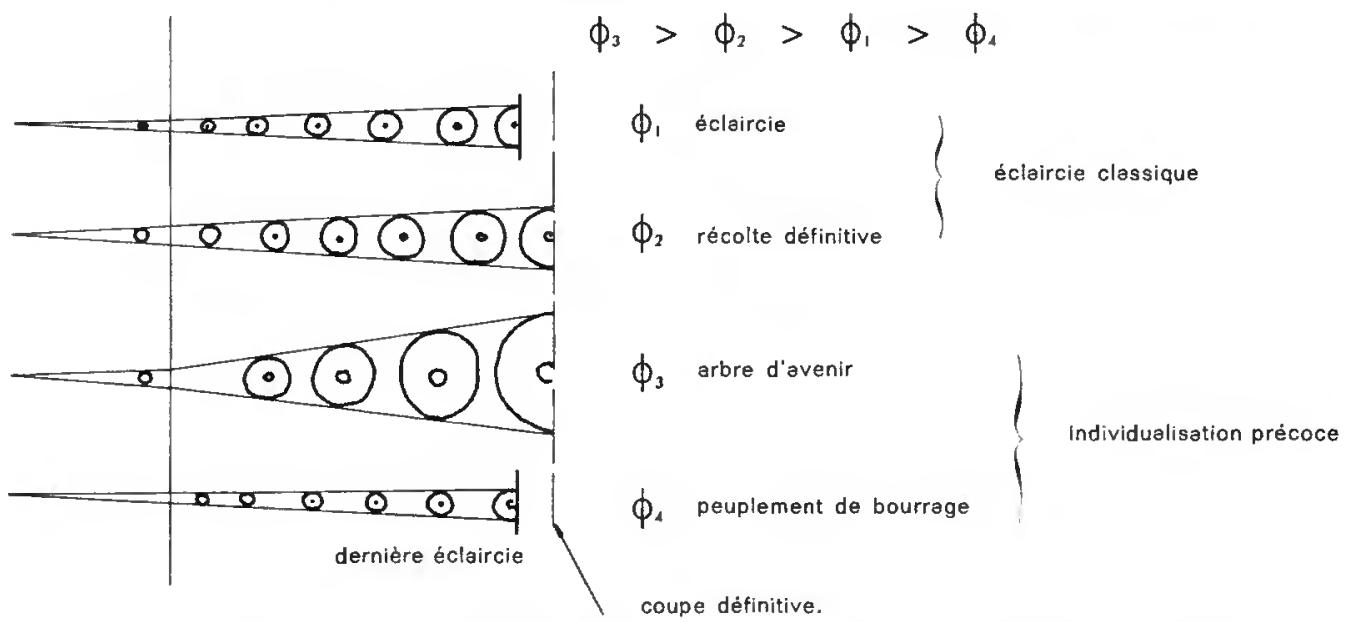

\section{SCHÉMA DE VARIATION AVEC L'AGE DU DIAMĖTRE DU HOUPPIER D'UN ARBRE DESTINÉ A L'ÉCLAIRCIE ET D'UN ARBRE DE COUPE DE RÉGĖNĖRATION}

\section{Objection: pas d'éclaircle rentable. Une grosse partie de la production d'avenir serait en bols sans valeur.}

Nous estimons que les éclaircies destinées à donner du bois de râperie ne seront pas commercialisables d'ici très peu d'années, par suite de la concurrence des bois étrangers qui peuvent être récoltés par grandes quantités dans des conditions de mécanisation excellente. Néanmoins, pour les poteaux de llgne, le problème serait peut-être un peu différent; il est à remarquer à ce sujet que les arbres de remplissage maintenus très serrés pendant longtemps pourront peut-être faire à la fin du peuplement d'excellents produits (principalement pour les résineux).

Pour ce qui est de la cime, comme il s'agit d'une coupe importante, une mécanisation serait peut-être possible pour le bois de râperie, toutes les cimes étant débitées en plaquettes sur coupe. Encore ne faut-il pas se faire trop d'illusions même si le houppier représente une partie importante de la hauteur totale de l'arbre.

Si l'on assimile l'arbre à un cône parfait, on obtient le tableau suivant:

\begin{tabular}{|c|c|c|c|c|}
\hline $\begin{array}{l}\text { Pourcentage des hauteurs des cimes } \\
\text { par rapport à la hauteur totale de } \\
\text { l'arbre } \ldots \ldots \ldots \ldots \ldots \ldots \ldots \ldots \ldots \ldots\end{array}$ & $50 \%$ & $55 \%$ & $60 \%$ & $65 \%$ \\
\hline $\begin{array}{l}\text { Pourcentage des volumes de cime } \\
\text { correspondants perdus ou de faible } \\
\text { valeur } \ldots \ldots \ldots \ldots \ldots \ldots \ldots \ldots \ldots\end{array}$ & $12,5 \%$ & $16,5 \%$ & $22 \%$ & $27,5 \%$ \\
\hline
\end{tabular}

Bien entendu, l'arbre est en première approximation intermédiaire entre le cylindre et le cône, mais ne prendre que $40 \%$ de la hauteur totale de l'arbre comme grume ne veut pas dire que $60 \%$ de la production soit perdue.

Que pourraient représenter les revenus des éclaircies pour le chêne dans la méthode traditionnelle jusqu'à 150 ans sachant que les premières éclaircies sont de faible diamètre pour une production à l'hectare de l'ordre de $6 \mathrm{~m}^{3} / \mathrm{ha} / \mathrm{an}$ ?

Dans la méthode envisagée, nous prévoyons a 150 ans la récolte définitive d'environ $200 \mathrm{~m}^{3}$ de bois de gros diametre et sans défaut qui pourront valoir :

$$
200 \mathrm{~m}^{3} \times 700 \mathrm{~F} / \mathrm{m}^{3}=140.000 \mathrm{~F} \text {. }
$$

Le fait de négliger la valeur des éclaircies pour parvenir plus vite à une vente de grumes de qualité nous semble justifié, la perte étant d'environ 8 à $21 \%$ pour un revenu final obtenu beaucoup plus rapidement. 


\begin{tabular}{|c|c|c|c|c|c|c|c|}
\hline \multirow{2}{*}{$\begin{array}{l}\text { Age du } \\
\text { peuplement }\end{array}$} & \multirow{2}{*}{$\begin{array}{l}\text { Nomb. de } \mathrm{m}^{3} \\
\text { retirés en } \\
\text { éclaircle } \\
\text { à partir } \\
\text { de } 70 \text { ans }\end{array}$} & \multirow{2}{*}{$\begin{array}{l}\text { Prix } \\
\text { du } m^{3}\end{array}$} & \multirow{2}{*}{$\begin{array}{l}\text { Bénéfice } \\
\text { dû aux } \\
\text { éclaircies }\end{array}$} & \multicolumn{2}{|c|}{$\begin{array}{l}\text { Actualisation a } \\
150 \text { ans avec un } \\
\text { taux de placement } \\
\text { de } 2 \%\end{array}$} & \multicolumn{2}{|c|}{$\begin{array}{c}\text { Actualisation a } \\
150 \text { ans avec un } \\
\text { taux de placement } \\
\text { de } 3 \%\end{array}$} \\
\hline & & & & $\begin{array}{l}\text { Coef. } \\
\text { multipli- } \\
\text { cateur }\end{array}$ & Bénéfice & $\begin{array}{l}\text { Coef. } \\
\text { multipli- } \\
\text { cateur }\end{array}$ & Bénéfice \\
\hline 70 & 30 & 0 & 0 & 4,875 & 0 & 10,641 & 0 \\
\hline 80 & 30 & 10 & 300 & 4,000 & 1.200 & 7,918 & 2.375 \\
\hline 90 & 35 & 15 & 525 & 3,281 & 1.723 & 5,892 & 3.094 \\
\hline 100 & 35 & 25 & 875 & 2,692 & 2.356 & 5,585 & 4.887 \\
\hline 110 & 35 & 35 & 1.225 & 2,208 & 2.705 & 3,959 & 4.850 \\
\hline 120 & 35 & 45 & 1.575 & 1,811 & 2.853 & 2,807 & 4.411 \\
\hline 130 & 40 & 65 & 2.600 & 1,486 & 3.864 & 1,806 & 4.696 \\
\hline \multirow[t]{2}{*}{140} & 40 & 90 & 3.600 & 1,219 & 4.389 & 1,344 & 4.839 \\
\hline & 280 & & 10.700 & & 19.090 & & 29.152 \\
\hline
\end{tabular}

\section{3 - 6 - A QUEL AGE COMMENCER A DEVELOPPER LE HOUPPIER ?}

L'âge auquel débute le " forçage " du houppier peut être approximativement prévu quand on dispose de tables de production.

Dans la table de production de Sologne de Decourt (R. F. F. décembre 1965), prenons pour exemple en classe 3 une parcelle de Pin sylvestre que l'on désire exploiter à 80 ans. La " hauteur potentielle " pourra être fixée égale à la moyenne des hauteurs moyennes et dominantes soit : $\frac{25,9+24,6}{2}=25,2$, on peut estimer qu'une hauteur de grume de $40 \%$ de la hauteur totale est souhaitable, soit environ $10 \mathrm{~m}$ (pour des pins de meilleure race, il doit être préférable de prendre $50 \%$ ).

Dans un peuplement jeune, il semble raisonnable de retenir $1 / 3$ de hauteur de cime pour $2 / 3$ de grumes sans que cela nuise à la croissance. II suffit donc de rechercher à quel âge l'arbre aura atteint $15 \mathrm{~m}$. La même moyenne que ci-desus donne 38 ans.

Jusqu'à 38 ans environ, l'arbre sera dégagé, mais sans excès pour ne pas développer de grosses branches, puis l'élagage artificiel sera complété jusqu'à $10 \mathrm{~m}$ et toute possibilité de développement sera donnée au houppier.

\footnotetext{
La moitiè supérieure du tronc présente des défauts, possède des vices apparents et est d'un diametre relativement foible. II aurait sans doute eté souhaitable d'y laisser des branches vives pour augmenter le diametre de la blile de pied, sa qualité et donc son prix, spécialement pour le hetre oú l'accroissement rapide est recherche.
}

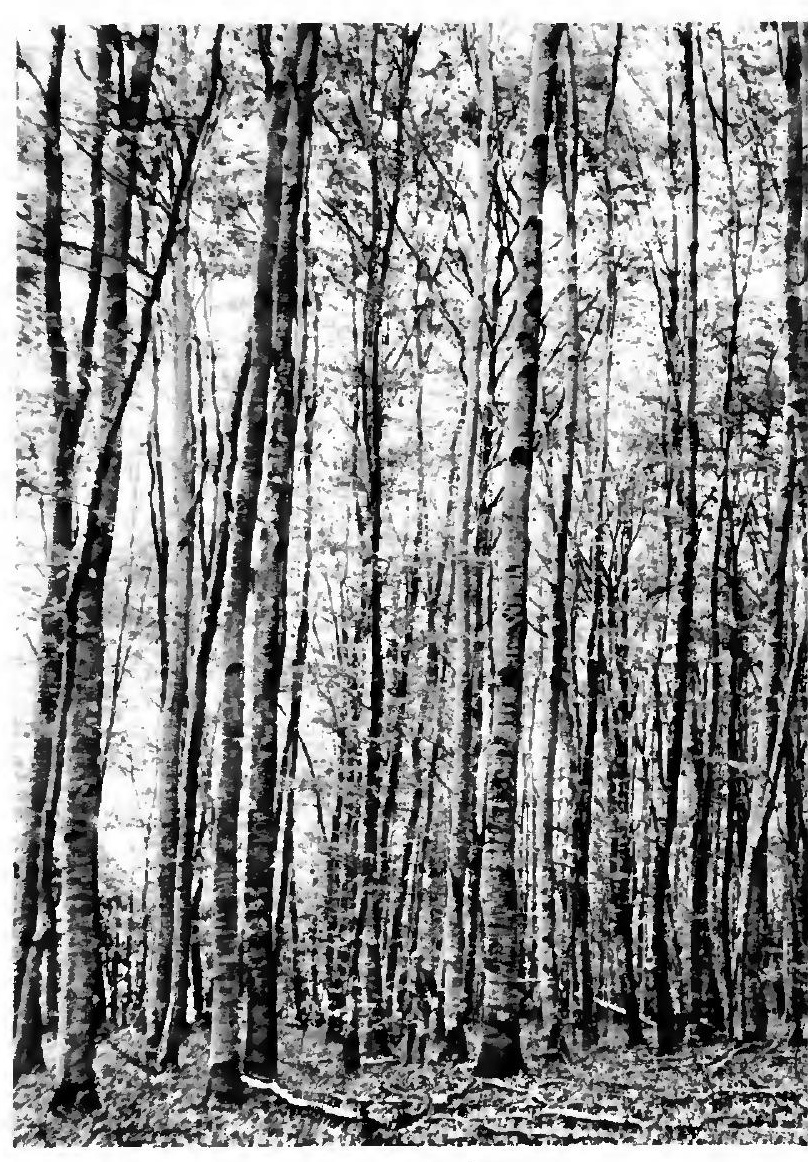

PHOTO TOUIGOUAT 
qu'il s'est fixées; Il les marque à la peinture. Les éclaircies consistent à couper une ou plusieurs tiges autour de cet arbre avec une rotation d'autant plus rapide qu'il réagit mieux aux traitements. Il faut néanmoins se garder * d'isoler * l'arbre d'avenir et le démarrage doit être progressif. Pour être efficaces, les éclaircies se feront donc à courte échéance dans les feuillus (tous les 5 ans par exemple durant les 15 premières années). II est bon de prendre alors la méthode utilisée à BERCE qui consiste à dire au marteleur de ne retirer qu'une, deux ou trois tiges par arbre d'avenir.

Les éclaircies sont relativement peu coûteuses; en effet, cela revient à couper 100 à 150 tiges par hectare sans façonnage. (II existe avec les produits chimiques un risque dû́ aux anastomoses de racines entre l'arbre d'avenir et ses voisins et il semble prudent de préférer la scie).

L'àge auquel la hauteur de grume est atteinte peut varier considérablement suivant les terr'ains. Pour le chêne, il semble facile de choisir parfois à 40 ans alors que dans d'autres cas le choix a 60 ans est encore aléatoire; pour les résineux, la sélection peut être beaucoup plus précoce d'autant plus que l'élagage artificiel pourra remédier facilement aux défauts puisque seuls sont élagués les arbres d'avenir et donc qu'une plus-value importante pour chacun d'eux en résultera.

\section{4-3 - ARBRES DE 25 A 30 ANS - PRESELECTION D'ARBRES D'AVENIR}

\section{$4-3$ - 1 - Feuillus}

Certains sylviculteurs sont d'avis de laisser les peuplements feuillus sans interventions jusqu'à 40 ans et plus. Dans ce cas nous pensons inutile de faire un choix d'arbres d'avenir auparavant. Par contre d'autres interviennent tôt dans le peuplement et en particulier de 25 à 30 ans. IIs se trouvent alors en face d'environ 10.000 tiges à l'hectare dans les bonnes régénérations et chacune de leur intervention peut conduire a couper 1.000 tiges ou plus par hectare.

Pour réduire le coût de ces interventions, nous sommes partisans d'une présélection des arbres d'avenir. A cet àge, dans de nombreux cas, il n'est pas possible de les choisir définitivement. Le sylviculteur choisit le nombre de tiges qui lui semble optimal par hectare. Supposons par exemple 50, correspondant à une largeur de cime future de 15 mètres. Ces nettoiements ne porteront qu'autour de ces 50 arbres c'est-à-dire qu'il n'aura à couper dans tout son nettoiement que 50 à 100 tiges. Cela lui permettra aussi de faire de "l'arboriculture ". II pourra, pour ces 50 arbres, faire éventuellement un raccourcissement de certaines branches trop vigoureuses sans pour autant faire un élagage aussi rigoureux que celui du peuplier. Bien entendu à cet àge il n'est pas question d'isoler ou même de desserrer les arbres. Simplement les arbres choisis seront conduits et surveillés, pour certains il n'y aura rien à retirer en éclaircie. La dépense du choix des arbres faite (il est délicat), les éclaircies sont économiques car elles conduisent à concentrer le travail sur 200 ou $300 \mathrm{~m}^{2}$ par hectare soit 2 a $3 \%$ de la surface totale.

Si aux environs de 40 à 60 ans le sylviculteur constate une erreur de choix, il peut alors changer son choix car jusqu’à cette époque tous les arbres ont été maintenus serrés et aucun ne s'est développé de manière trop priviléglée; tout au plus les arbres indiqués sont-ils légèrement plus forts.

Là encore, on ne saurait trop recommander de donner des normes de dégagements aux ouvriers qui ne doivent pas retirer plus de deux ou trois petites ou une ou deux grossas tlges par arbre d'avenir avec tout ce que cela comporte comme inconvénients. Manquer de prudence à cet égard pourrait conduire à faire plus de mal que de bien.

\section{4-3-2 - Plantation résineuse}

Vu la courte durée de vie du peuplement (60 à 100 ans) et les possibilités d'élagage, il importe de faire une sélection précoce et la présélection se fait plus jeune (15 à 20 ans) ou même peut n'être pas nécessaire, la sélection définitive pouvant se faire dès que le peuplement devient facilement pénétrable. Plus la durée de vie est courte, plus la sélection doit étre précoce.

\section{4-4 - REgenERATION ACQUISE DE 5 A 15 ANS}

\section{4-4-1 - Premler cas: fourré dont les tlges ne dépassent pes $2 \mathrm{~cm}$ de dlametre}

Supposons un fourré bien dense d'une régénération bien venante. Néanmoins certaines essences indésirables sont mêlées au reste du peuplement. Les nettoiements traditionnels conduisent à traiter toute la surface du peuplement. Une grande partie du temps de l'ouvrier consiste à se frayer un chemin et à lutter contre ronces et chèvrefeuille. II coupe plusieurs milliers de tiges par hectare. 
On peut se demander jusqu'a quel point une intervention aussi intensive est rentable. Prenons le cas pour préciser les choses d'une chênaie pédonculée à charme. Dès l'ouverture du peuplement, les charmes se sont régénérés et le chêne n'a suivi que plus tard, donnant une régénération touffue de charmes émaillée de chênes que nous supposerons bien répartis.

La méthode consiste à ouvrir dans la parcelle, tous les $10 \mathrm{~m}$ environ, un passage au gyrobroyeur.

Après avoir primitivement opté pour un véritable quadrillage, nous avons abandonné cette méthode car elle double le travail du gyrobroyeur, augmente les effets de bordure et diminue la possibilité de sélection dans les mauvaises régénérations où l'essence noble est rare (tache de charme avec de rares chênes).

L'ouverture des bandes au gyrobroyeur est peu coûteuse et le travail est rapide.

Le drainage (fossés ouverts par cureuse rotative) passera de prélérence par ces passages ouverts en conséquence. L'ouvrier, de part et d'autre de ce passage, à 1,50 m environ à l'intérieur du peuplement, dégagera tous les $2 \mathrm{~m}$ environ les plants de chêne à protéger. Ceci conduira à sauver sur le terrain environ 1.000 chênes répartis en lignes distantes d'environ 5 m. Il est bien entendu que d'autres chênes subsisteront et que d'autres qui auraient été sauvés par des dégagements périront mais cela permet de faire descendre de manière importante le prix de revient du dégagement à l'hectare.

Les régénérations de ce genre ne sont pas rares oủ le prix de revient des dégagements est monté à 2.000 ou $3.000 \mathrm{~F}$ par hectare pour des résultats moyens. II convient de souligner que l'ouvrier n'agit pas forcément partout mais seulement là où son intervention peut être efficace. Si le gyrobroyeur traverse une tache de chêne pur l'ouvrier n'aura strictement rien à laire. Par contre, dans une tache de charme pur, l'unique plant de chêne qui était dominé fera l'objet de soins attentifs et progressifs. L'ouvrier pourra même lutter contre le chèvrefeuille là où il est particulièrement abondant et menace les tiges choisies.

Là encore, il convient d'empêcher l'ouvrier de faire trop de travail. Il n'est pas question d'isoler les plants, il est simplement nécessaire de leur permettre de s'étoffer.

Le dépressage de régénération naturelle de résineux ou de semis de pins (maritime) peut se faire suivant la méme méthode à peu de frais.

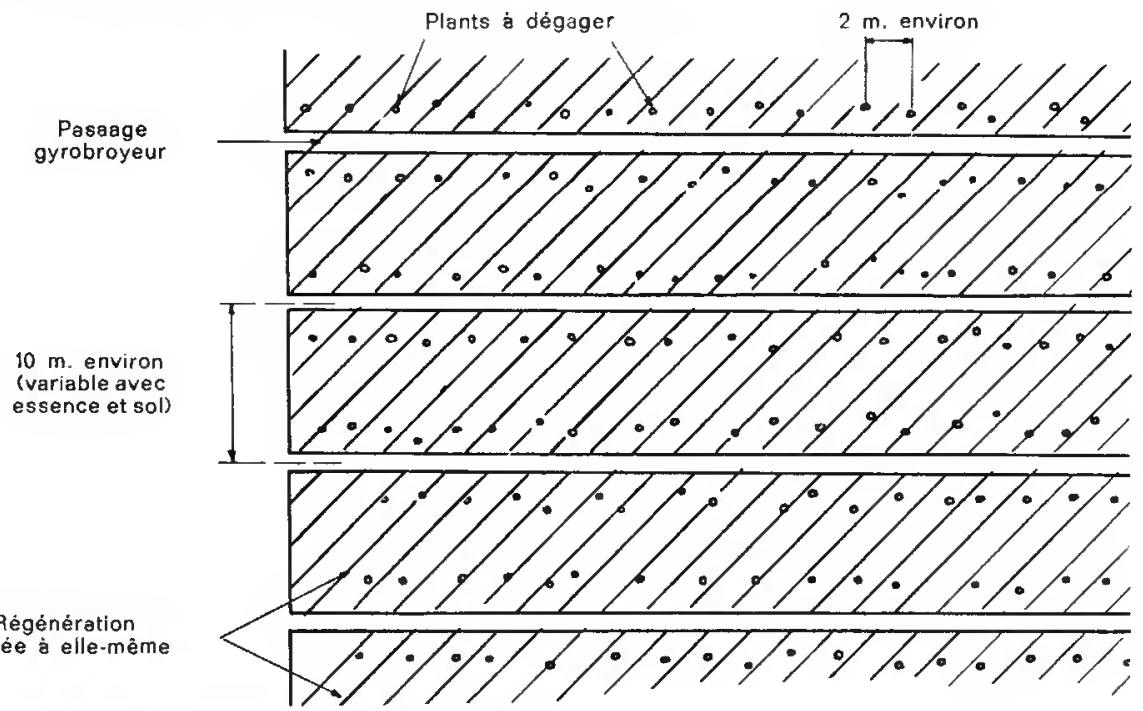

\section{4-4-2 - Deuxjème cas : fourré gaulls de 10-15 ans}

Au-dessus d'un certain diamètre, seuls certains types de débroussailleuses peuvent être utilisés mais les gaulis risquent de a verser ». Il semble plus intéressant alors d'ouvrir des passages tıès étroits qui permettent à l'ouvrier d'approcher les plants. Là encore, deux cas sont possibles: prendre les plants à $1,50 \mathrm{~m}$ ou $2 \mathrm{~m}$ à droite et à gauche de ce chemin d'accès et ne faire ce chemin de 50 à $60 \mathrm{~cm}$ de large que tous les $10 \mathrm{~m}$ environ, soit prendre les arbres au bord même du chemin et faire les chemins plus rapprochés.

Dés que le peuplement a trois mètres de hauteur, les cimes se touchent et nous pensons qu'il n'y aura pas trop de difficultés à entretenir le chemin au moyen par exemple de débroussailleuse portative ou en badigeonnant les chicots au P. 80. 
Nous proposons donc:

$1^{\circ}$ - d'éliminer les réserves incapables d'attendre la régénération ;

$2^{\circ}$ - de choisir sur le terrain les meilleures parmi les subsistantes et d'estimer pour chacune la capacité de développement du houppier en 70 ans;

$3^{a}$ - dans les vides en réserves où les houppiers des réserves ne pourront se rejoindre, choisir des baliveaux ou des brins de taillis et estimer leur possibilité de développement sachant que la grume recherchée doit être très courte pour pouvoir être de gros diamètre. La longueur de grume du taillis recherchée pourrait être inférieure à celle des réserves.

Le calcul sera fait comme pour une futaie, sachant qu'en 80 ans on veut obtenir des produits vendables et compte tenu du fait que les rejets poussent plus vite que les arbres de franc pied dans leurs premières années. Il est préfćrable, nous semble-t-il, d'obtenir une grume courte et de gros diamètie plutôt qu'une magnifique grume bien élancèe de 25 ou $30 \mathrm{~cm}$ de diamètre en fin de révolution.

Bien entendu, le taillis sera maintenu sur pied. II jouera le rôle de peuplement de bourrage.

\section{4-7 - ENRÉSINEMENT DE FUTAIES FEUILLUES}

Dans un même ordre d'idées, si une futaie feuillue peut se régénérer facilement avec des essences d'intérêt secondaire (charme, ...), il pourrait être intéressant de planter des lignes de résineux (tous les 10 mètres pour le pin) parmi une régénération feuillue, qui seules seraient dégagées avec ou sans l'aide de passages au gyrobroyeur avec individualisation précoce des arbres d'avenir et conduite du peuplement comme il est décrit précédemment.

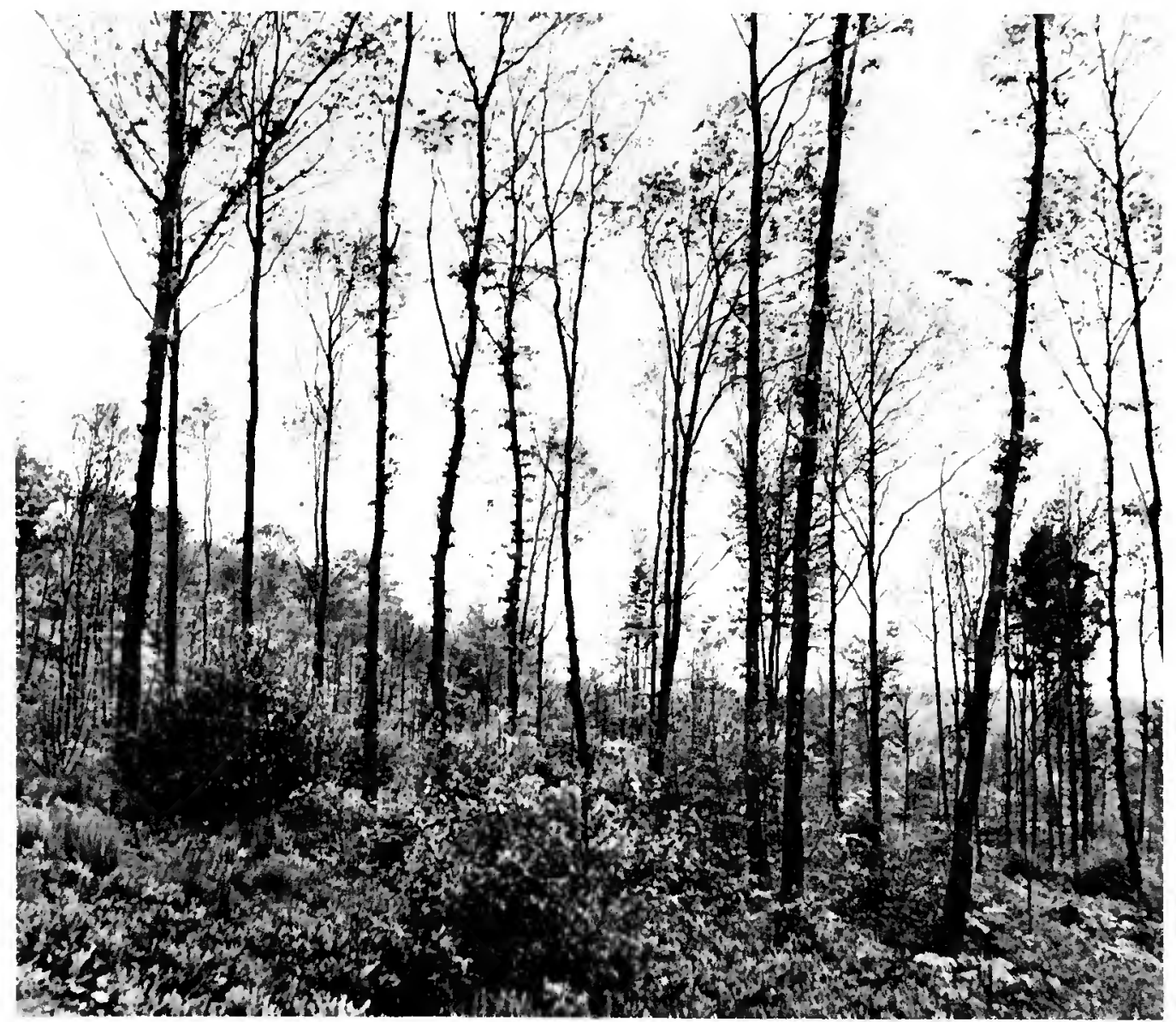

Balivage de taillis-sous-futaie.

Arbres surexposés après avolr été étouffés, pousse de gourmands qui dèpréclent la grume, brancha basse morte, après avoir acquis un diamètre souvent suffisant pour étre noclf (nceuds noirs) 


\section{4-8 - REMARQUES SUR L'ÉLAGAGE ARTIFICIEL}

La mise en place de la méthode va de pair avec une généralisation de l'élagage artificiel. Dans les futaies feuillues, le peuplement sera de toutes façons maintenu fermé durant les premières années pour tavoriser l'élagage naturel. Mais lors du développement du houppier, il pourra être utile d'aider artificiellement l'élagage des branches les plus hautes en passe d'être éliminées naturellement, ceci permettra de gagner quelques années sur le développement du houppier et donc de la grume.

Pour le résineux, l'élagage des sujets d'avenir se fera au moment de leur choix et servira à leur repérage.

Si dans un peuplement de résineux à sylviculture de type traditionnel on peut s'interroger sur l'économie de l'élagage, puisqu'on ne sait si les tiges élaguées partíront ou non en éclaircie, dans le cas d'arbres d'avenir choisis précocement, tous les arbres élagués doivent donner des grumes et l'investissement est sûr.

Dans le cas des feuillus, l'élagage mécanique pourra éventuellement être complété d'un élagage chimique au cas oủ des rejets apparaîtraient (et si ces méthodes s'avèrent au polnt).

La photo ci-contre d'un peuplement de pins Weymouth montre tout l'intérêt qu'il pourra en résulter pour les grumes d'avenir.

\section{5 - LES AVANTAGES DE LA MÉTHODE}

La méthode de choix d'arbres d'avenir et en particulier celle faisant l'objet de cet article présente un certain nombre d'avantages. Ils n'interviennent pas tous au même titre et au même degrè pour toutes les essences, mais il nous a semblé utile de les rassembler ci-après:

\section{5-1 - REGENERATION}

- Possibilité de bonne sélection génétique à bas prix.

- Excellente utilisation des vergers à graines qui deviennent plus rentables et donc en extension plus rapide.

- Utilisation d'engrais concentré à la plantation sur une petite surface utile par hectare.

- La régénération du chêne se fait plus rapidement et sans trop d'à-coups; elle est moins soumise à la glandée décennale; le sol plus rapidement couvert se dégrade moins.

- Nettoiements, dépressages et éclaircies des peuplements jeunes relativement intensifs et peu coûteux.

\section{5-2 - SYLVICULTURE - AMELIORATION DES PEUPLEMENTS}

- Sélection méticuleuse des arbres (une fois pour toutes).

- Densité régulière. Il est difficile dans le cas de traitements en futaie ordinaire de passer d'une densité régulière de 300 arbres par hectare à une densité de 250 arbres, à la fin du peuplement.

- Enracinement puissant (résistance au vent).

- Pas de passage d'engins dans les coupes d'éclaircies, donc pas de tassement du sol avec dégradation de la structure; moins de détérioration aux arbres.

- Facilité relative de traitement de futaie mélangée d'essences de longévité différente (chêne, hêtre) par raccourcissement de la révolution.

- Enrésinement de régénération feuillue plus aisée et moins coûteuse.

- Dans le cas de plantations de résineux avec bourrage feuillus :

- les risques d'attaque (bostryche, champignons), sont moindres (rouille du Weymouth).

- le sol est maintenu en bon état, ce qui permet plusieurs végétations successives de résineux sans diminution de rendements.

- les risques d'incendie sont limités.

\section{5-3 - TECHNOLOGIE - ECONOMIE}

- Martelages moins coûteux car plus rapides.

- Possibilités d'élagage économique.

- Eclaircies laissant les bois à terre, donc peu coûteuses. Leur période peut donc étre courte et le développement des houppiers d'arbres d'avenir progressif et rapide. Donc pas d'arbres 
" étouffés "par des périodes de 15 ans et pas de descente de cime due à des éclaircies fortes sur tiges serrées.

- Suppression des unités nuisibles de 2 ou 3 arbres maintenus serrés en fin de révolution.

- Houppiers symétriques et cœurs centrés.

- Cernes réguliers et importants conduisant précocement à de grosses grumes.

- Obtention de grumes intéressantes avec las rejets de taillis-sous-futaie en vieillissement.

- Rotation plus rapide des capitaux, augmentation du taux de placement.

\section{5 - 4 - POSSIBILITES NOUVELLES}

- Posslbllités de sylviculture des frêne, fruitiers, érable, noyer, etc.

\section{6 - CONCLUSION}

Cette liste d'avantages ne fait qu'eftleurer les raisons réelles qui militent en faveur de l'indivldualisation précoce des arbres d'avenir dans une sylviculture moderne.

Pour assurer la pérennité de la forêt productrice de bois, la longueur des révolutions doit être réduite et l'importance du capital investi diminuée.

Dans un monde dont l'économie est en accélération permanente, les íncertitudes sur les placements à long terme sont de plus en plus pesantes. Peut-on alors faire confiance à l'avenir ef travailler sans se soucier des fluctuations du moment ou de l'époque ? II a fallu deux siècles pour que les bois de marine de Colbert changent de destination. L'Histoire a bien voulu être clémente et les bois de marine donnent un excellente tranche. Le sera-t-elle la prochaine fois ? Quand sera cette prochaine fois? II y a tout lieu de penser qu'il ne s'agira plus de siècles mais de décennies, et les forestiers n'auront peui-être pas à chaque lois la même chance.

Que croire alors ? Sur quel critère s'appuyer pour croire dans le placement forestier?

Nous persistons à penser que du point de vue technologique, une grosse grume sans dẻfaut sera toujours demandée. Par ailleurs, un arbre de 150 ans ou plus sera du fait même de son âge un produit de luxe car long à obtenir. Il gardera donc une valeur importante, pour l'urbanisme, le tourisme ou tout autre raison, en plus de ses usages traditionnels.

II nous semble donc raisonnable de persister à planter des arbres à longue révolution.

Mais dans ce monde tournant sans cesse plus vite, il faut néanmoins s'adapter. "Les siècles raccourcissent " et il semble souhaitable aussi de raccourcir autant que possible les révolutions des arbres. La génétique et les façons culturales ont permis de raccourcir de près d'un tiers la durée de vie des peupliers. Pourquoi le chéne et le hêtre seraient-ils * tabous n? Qui prouve que le cerne fin du chêne demeurera la panacée? Rien ne nous semble s'opposer a priori à une croissance accélérée des bois.

Si le propriétaire est intéressé par une révolutlon plus courte, il l'est aussi pour les essences forestières longévives, par la réduction du capltal à investir à l'hectare, qui sauf mécanisation, ne cessera de monter. Les opérations manuelles coûteront probablement 4 à 5 fois plus cher d'ici 50 ans.

L'ensemble de la chaîne de production doit être mécanisée dans un ensemble cohérent.

La régénération naturelle forestière qui reste souhaitable dans de nombreux cas est peut-être condamnée à terme. Les compléments de régénération manuels étant appelés à devenir prohibitifs.

La méthode d'individualisation précoce des arbres d'avenir essaye de réduire la révolution des essences forestières tout en réduisant dans la mesure du possible l'emploi de la main-d'cuvre. Elle substitue à la notion de sylviculture de peuplement celle d'individus.

Marc de SAINT-VAULAY

Ingénieur du G. R. E. F. à I'O. N. F. Chef du Centre de Gestion de Bourges

2. Place de la Préfecture 18 - BOURGES 